\title{
FELLER SEMIGROUPS GENERATED BY DEGENERATE ELLIPTIC OPERATORS*
}

\author{
K. Taira, A. Favini, And S. Romanelli
}

\begin{abstract}
This paper is devoted to the functional analytic approach to the problem of construction of Feller semigroups with sticky boundary condition on various spaces of continuous functions, generalizing the previous work. More precisely we construct Feller semigroups corresponding to such a diffusion phenomenon that a Markovian particle moves continuously in the state space, sticking to the boundary.
\end{abstract}

\section{Introduction and Results}

Let $D$ be a bounded domain of Euclidean space $\mathbf{R}^{N}, N \geq 2$, with smooth boundary $\partial D$. In this paper we consider a second-order, degenerate elliptic differential operator $A$ with real coefficients of the form

$$
A u(x)=\sum_{i, j=1}^{N} a^{i j}(x) \frac{\partial^{2} u}{\partial x_{i} \partial x_{j}}(x)+\sum_{i=1}^{N} b^{i}(x) \frac{\partial u}{\partial x_{i}}(x)+c(x) u(x) .
$$

Here:

(1) $a^{i j}(x) \in C^{\infty}\left(\mathbf{R}^{N}\right), a^{i j}(x)=a^{j i}(x), x \in \mathbf{R}^{N}$, and

$$
\sum_{i, j=1}^{N} a^{i j}(x) \xi_{i} \xi_{j} \geq 0, \quad x \in \mathbf{R}^{N}, \xi \in \mathbf{R}^{N} .
$$

(2) $b^{i}(x) \in C^{\infty}\left(\mathbf{R}^{N}\right)$.

(3) $c(x) \in C^{\infty}\left(\mathbf{R}^{N}\right)$ and $c(x) \leq 0$ in $D$.

In order to state our fundamental hypothesis for $A$, we introduce a function $b\left(x^{\prime}\right)$ on the boundary $\partial D$ by the formula (see [4])

$$
b\left(x^{\prime}\right)=\sum_{i=1}^{N}\left(b^{i}\left(x^{\prime}\right)-\sum_{j=1}^{N} \frac{\partial a^{i j}}{\partial x_{j}}\left(x^{\prime}\right)\right) n_{i}, \quad x^{\prime} \in \partial D,
$$

where $\mathbf{n}=\left(n_{1}, n_{2}, \ldots, n_{N}\right)$ is the unit interior normal to the boundary $\partial D$ at $x^{\prime}$. The function $b\left(x^{\prime}\right)$ is called the Fichera function for the operator $A$. We divide the boundary $\partial D$ into the following four disjoint subsets (cf. [4], [6], [7]):

$$
\Sigma_{3}=\left\{x^{\prime} \in \partial D: \sum_{i, j=1}^{N} a^{i j}\left(x^{\prime}\right) n_{i} n_{j}>0\right\} .
$$

*Work partially supported by M.U.R.S.T. $60 \%$ and $40 \%$ under the auspices of G.N.A.F.A. of C.N.R.

Typeset by $\mathcal{A} \mathcal{M} \mathcal{S}-\mathrm{T}_{\mathrm{E}} \mathrm{X}$ 


$$
\begin{aligned}
& \Sigma_{2}=\left\{x^{\prime} \in \partial D: \sum_{i, j=1}^{N} a^{i j}\left(x^{\prime}\right) n_{i} n_{j}=0, b\left(x^{\prime}\right)<0\right\} . \\
& \Sigma_{1}=\left\{x^{\prime} \in \partial D: \sum_{i, j=1}^{N} a^{i j}\left(x^{\prime}\right) n_{i} n_{j}=0, b\left(x^{\prime}\right)>0\right\} . \\
& \Sigma_{0}=\left\{x^{\prime} \in \partial D: \sum_{i, j=1}^{N} a^{i j}\left(x^{\prime}\right) n_{i} n_{j}=0, b\left(x^{\prime}\right)=0\right\} .
\end{aligned}
$$

Then our hypothesis for the operator $A$ is stated as follows:

(H) $\partial D=\Sigma_{2} \cup \Sigma_{3}$ and each set $\Sigma_{i}, i=2,3$, consists of a finite number of connected hypersurfaces.

It is worth pointing out here (see [6], [7], [8]) that we may impose a boundary condition only on the set $\Sigma_{2} \cup \Sigma_{3}$, since a Markovian particle reaches the boundary $\partial D$ by means of the diffusion vector fields

$$
\pm X_{i}(x)= \pm \sum_{j=1}^{N} a^{i j}(x) \frac{\partial}{\partial x_{j}}, \quad 1 \leq i \leq N
$$

and the drift vector field

$$
X_{0}(x)=\sum_{i=1}^{N}\left(b^{i}(x)-\sum_{j=1}^{N} \frac{\partial a^{i j}}{\partial x_{j}}(x)\right) \frac{\partial}{\partial x_{i}} .
$$

Now let $C(\bar{D})$ be the Banach space of real-valued, continuous functions on the closure $\bar{D}=D \cup \partial D$, equipped with the maximum norm

$$
\|f\|=\max _{x \in \bar{D}}|f(x)|, \quad f \in C(\bar{D}) .
$$

A strongly continuous semigroup $\left\{T_{t}\right\}_{t \geq 0}$ on the space $C(\bar{D})$ is called a Feller semigroup if it is nonnegative and contractive on $C(\bar{D})$, that is,

$$
f \in C(\bar{D}), 0 \leq f(x) \leq 1 \quad \text { on } \bar{D} \Longrightarrow 0 \leq T_{t} f(x) \leq 1 \quad \text { on } \bar{D}
$$

It is known (see [8]) that if $\left\{T_{t}\right\}_{t \geq 0}$ is a Feller semigroup on $C(\bar{D})$, then there exists a unique Markov transition function $p_{t}(x, \cdot)$ on $\bar{D}$ such that

$$
T_{t} f(x)=\int_{\bar{D}} p_{t}(x, d y) f(y), \quad f \in C(\bar{D})
$$

Furthermore, it can be shown that the function $p_{t}(x, \cdot)$ is the transition function of some strong Markov process; hence the value $p_{t}(x, E)$ expresses the transition probability that a Markovian particle starting at position $x$ will be found in the set $E$ at time $t$. 
The purpose of this paper is to construct Feller semigroups on various spaces of continuous functions on the closure $\bar{D}$ corresponding to such a diffusion phenomenon that a Markovian particle moves continuously in the state space $\bar{D}$ sticking to the boundary, which generalizes the previous works Clément-Timmermanns [1] and Taira [10].

Our starting result is the following generalization of [1, Theorem 2] to the multidimensional case (see also [3, Theorem 2.2] in the framework of analytic semigroups):

Theorem 1. Assume that the operator A satisfies hypotheses (H). Then there exists a Feller semigroup $\left\{T_{t}\right\}_{t \geq 0}$ on $C(\bar{D})$ whose infinitesimal generator $\mathcal{A}$ is characterized as follows:

(1) The domain $D(\mathcal{A})$ of $\mathcal{A}$ is the space

$$
D(\mathcal{A})=\{u \in C(\bar{D}): A u \in C(\bar{D}), A u=0 \text { on } \partial D\} .
$$

(2) $\mathcal{A} u=A u, u \in D(\mathcal{A})$.

Here $A u$ is taken in the sense of distributions.

We emphasize that the operator $A$ may be degenerate in the interior $D$, while Clément-Timmermanns [1] and Favini-Romanelli [3] consider the case where the operator $A$ is degenerate at the boundary $\partial D$, but is not degenerate (elliptic) in the interior $D$.

Rephrased, Theorem 1 asserts that we can construct a Feller semigroup corresponding to such a diffusion phenomenon that the viscosity phenomenon occurs at each point of the boundary. Indeed, it suffices to note that the boundary condition

$$
A u=0 \quad \text { on } \partial D
$$

is supposed to correspond to the viscosity phenomenon.

Secondly, we introduce a subspace of $C(\bar{D})$ as follows:

$$
C_{\pi}(\bar{D})=\{u \in C(\bar{D}): u \text { is constant on } \partial D\}
$$

The next theorem asserts the existence of a Feller semigroup on the space $C_{\pi}(\bar{D})$ :

Theorem 2. Assume that the operator A satisfies hypotheses (H). Then there exists a Feller semigroup $\left\{S_{t}\right\}_{t \geq 0}$ on $C_{\pi}(\bar{D})$ whose infinitesimal generator $\mathcal{P}$ is characterized as follows:

(1) The domain $D(\mathcal{P})$ of $\mathcal{P}$ is the space

$$
D(\mathcal{P})=\left\{u \in C_{\pi}(\bar{D}): A u \in C_{\pi}(\bar{D}), A u=0 \text { on } \partial D\right\} .
$$

(2) $\mathcal{P} u=A u, u \in D(\mathcal{P})$.

Rephrased, Theorem 2 asserts that we can construct a Feller semigroup corresponding to such a diffusion phenomenon that a Markovian particle sticks the boundary forever when it hits there for the first time.

Thirdly we introduce a subspace of $C_{\pi}(\bar{D})$ as follows:

$$
C_{0}(\bar{D})=\{u \in C(\bar{D}): u=0 \text { on } \partial D\} .
$$

The next theorem asserts the existence of a Feller semigroup on the space $C_{0}(\bar{D})$ : 
Theorem 3. Assume that the operator A satisfies hypotheses (H). Then there exists a Feller semigroup $\left\{U_{t}\right\}_{t \geq 0}$ on $C_{0}(\bar{D})$ whose infinitesimal generator $\mathcal{C}$ is characterized as follows:

(1) The domain $D(\mathcal{C})$ of $\mathcal{C}$ is the space

$$
D(\mathcal{C})=\left\{u \in C_{0}(\bar{D}): A u \in C_{0}(\bar{D})\right\} .
$$

(2) $\mathcal{C} u=A u, u \in D(\mathcal{C})$.

Rephrased, Theorem 3 asserts that we can construct a Feller semigroup corresponding to such a diffusion phenomenon that a Markovian particle is absorbed at the first moment when it hits the boundary.

Summing up, we have the following commutative diagram:

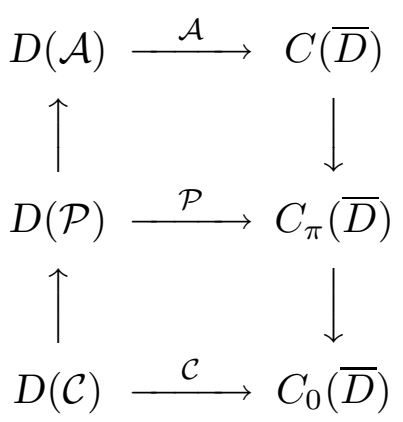

We remark that, in the one-dimensional case, Theorems 2 and 3 may be also compared with [3, Theorems 2.3 and 2.1], respectively, where the analyticity of the semigroups $\left\{S_{t}\right\}_{t \geq 0}$ and $\left\{U_{t}\right\}_{t \geq 0}$ is investigated.

The proof of Theorems 1, 2 and 3 will be carried out in Section 3, by following the proof of [10, Main Theorem]. In Section 2 we present a brief description of the basic definitions and results about a class of semigroups, Feller semigroups, associated with Markov processes in probability theory, which forms a functional analytic background for the proof of Theorems 1, 2 and 3.

The authors are grateful to the referee for his careful reading of the manuscript and many valuable suggestions. A major part of this work was done at the University of Bari in May, 1997 in the course of the JSPS-CNR exchange program. The research of the first author is partially supported by Grant-in-Aid for General Scientific Research (No. 10440050), Ministry of Education, Science and Culture of Japan.

\section{Theory of Feller Semigroups}

This section provides a brief description of the basic definitions and results about Feller semigroups, which forms a functional analytic background for the proof of Theorems 1, 2 and 3 in Section 3. The results discussed here are adapted from Dynkin [2], Lamperti [5] and also Taira [8].

2.1 Markov transition functions and Feller semigroups. Let $(K, \rho)$ be a locally compact, separable metric space and $\mathcal{B}$ the $\sigma$-algebra of all Borel sets in $K$.

A function $p_{t}(x, E)$, defined for all $t \geq 0, x \in K$ and $E \in \mathcal{B}$, is called a (temporally homogeneous) Markov transition function on $K$ if it satisfies the following four conditions: 
(a) $p_{t}(x, \cdot)$ is a nonnegative measure on $\mathcal{B}$ and $p_{t}(x, K) \leq 1$ for each $t \geq 0$ and $x \in K$.

(b) $p_{t}(\cdot, E)$ is a Borel measurable function for each $t \geq 0$ and $E \in \mathcal{B}$.

(c) $p_{0}(x,\{x\})=1$ for each $x \in K$.

(d) (The Chapman-Kolmogorov equation) For any $t, s \geq 0, x \in K$ and $E \in \mathcal{B}$, we have the formula

$$
p_{t+s}(x, E)=\int_{K} p_{t}(x, d y) p_{s}(y, E)
$$

Here is an intuitive way of thinking about the above definition of a Markov transition function. The value $p_{t}(x, E)$ expresses the transition probability that a physical particle starting at position $x$ will be found in the set $E$ at time $t$. Equation (2.1) expresses the idea that a transition from the position $x$ to the set $E$ in time $t+s$ is composed of a transition from $x$ to some position $y$ in time $t$, followed by a transition from $y$ to the set $E$ in the remaining time $s$; the latter transition has probability $p_{s}(y, E)$ which depends only on $y$. Thus a particle "starts afresh"; this property is called the Markov property.

We add a point $\partial$ to $K$ as the point at infinity if $K$ is not compact, and as an isolated point if $K$ is compact; so the space $K_{\partial}=K \cup\{\partial\}$ is compact.

Let $C\left(K_{\partial}\right)$ be the Banach space of real-valued, continuous functions on $K_{\partial}$ with the maximum norm

$$
\|f\|=\max _{x \in K_{\partial}}|f(x)|
$$

and introduce a closed subspace of $C\left(K_{\partial}\right)$ as follows:

$$
C_{0}(K)=\left\{f \in C\left(K_{\partial}\right): f(\partial)=0\right\}
$$

Then it is easy to see that

$$
C_{0}(K)=\left\{f \in C(K): \lim _{x \rightarrow \partial} f(x)=0\right\}
$$

Now we introduce some conditions on the measures $p_{t}(x, \cdot)$ related to continuity in $x \in K$, for fixed $t \geq 0$.

A Markov transition function $p_{t}(x, \cdot)$ is called a Feller function if the function

$$
T_{t} f(x)=\int_{K} p_{t}(x, d y) f(y)
$$

is a continuous function of $x \in K$ whenever $f$ is in $C(K)$, or equivalently, if we have

$$
f \in C(K) \Longrightarrow T_{t} f \in C(K) .
$$

We say that $p_{t}(x, \cdot)$ is a $C_{0}$-function if the space $C_{0}(K)$ is an invariant subspace of $C(K)$ for the operators $T_{t}$ :

$$
f \in C_{0}(K) \Longrightarrow T_{t} f \in C_{0}(K) .
$$


The Feller or $C_{0}$-property deals with continuity of a Markov transition function $p_{t}(x, \cdot)$ in $x$, and does not, by itself, have no concern with continuity in $t$. We give a necessary and sufficient condition on $p_{t}(x, \cdot)$ in order that its associated operators $\left\{T_{t}\right\}_{t \geq 0}$ be strongly continuous in $t$ on the space $C_{0}(K)$.

A Markov transition function $p_{t}(x, \cdot)$ on $K$ is said to be uniformly stochastically continuous on $K$ if the following condition is satisfied: For each $\varepsilon>0$ and each compact $E \subset K$, we have

$$
\lim _{t \downarrow 0} \sup _{x \in E}\left[1-p_{t}\left(x, U_{\varepsilon}(x)\right)\right]=0
$$

where $U_{\varepsilon}(x)=\{y \in K: \rho(x, y)<\varepsilon\}$ is an $\varepsilon$-neighborhood of $x$.

Then we have the following result (see [8, Theorem 9.2.3]):

Theorem 2.1. Let $p_{t}(x, \cdot)$ be a $C_{0}$-transition function on $K$. Then the associated operators $\left\{T_{t}\right\}_{t \geq 0}$, defined by the formula

$$
T_{t} f(x)=\int_{K} p_{t}(x, d y) f(y), \quad f \in C_{0}(K)
$$

are strongly continuous in $t$ on $C_{0}(K)$ if and only if $p_{t}(x, \cdot)$ is uniformly stochastically continuous on $K$ and satisfies the following condition:

(L) For each $s>0$ and each compact $E \subset K$, we have the assertion

$$
\lim _{x \rightarrow \partial} \sup _{0 \leq t \leq s} p_{t}(x, E)=0
$$

A strongly continuous semigroup $\left\{T_{t}\right\}_{t \geq 0}$ on the space $C_{0}(K)$ is called a Feller semigroup on $K$ if it is nonnegative and contractive on $C_{0}(K)$ :

$$
f \in C_{0}(K), 0 \leq f(x) \leq 1 \quad \text { on } K \Longrightarrow 0 \leq T_{t} f(x) \leq 1 \quad \text { on } K
$$

The next theorem gives a characterization of Feller semigroups in terms of Markov transition functions (see [8, Theorem 9.2.6]):

Theorem 2.2. If $p_{t}(x, \cdot)$ is a uniformly stochastically continuous $C_{0}$-transition function on $K$ and satisfies condition $(L)$, then its associated operators $\left\{T_{t}\right\}_{t \geq 0}$ form a Feller semigroup on $K$.

Conversely, if $\left\{T_{t}\right\}_{t \geq 0}$ is a Feller semigroup on $K$, then there exists a uniformly stochastically continuous $C_{0}$-transition $p_{t}(x, \cdot)$ on $K$, satisfying condition $(L)$, such that formula (2.2) holds.

2.2 Generation theorems of Feller semigroups. The next theorem is a version of the Hille-Yosida theorem adapted to the present context (see [8, Theorem 9.3.1 and Corollary 9.3.2]):

Theorem 2.3. (i) Let $\left\{T_{t}\right\}_{t \geq 0}$ be a Feller semigroup on $K$ and $\mathfrak{A}$ its infinitesimal generator. Then we have the following:

(a) The domain $D(\mathfrak{A})$ is dense in the space $C_{0}(K)$. 
(b) For each $\alpha>0$, the equation $(\alpha I-\mathfrak{A}) u=f$ has a unique solution $u$ in $D(\mathfrak{A})$ for any $f \in C_{0}(K)$. Hence, for each $\alpha>0$, the Green operator $(\alpha I-\mathfrak{A})^{-1}$ : $C_{0}(K) \rightarrow C_{0}(K)$ can be defined by the formula

$$
u=(\alpha I-\mathfrak{A})^{-1} f, \quad f \in C_{0}(K) .
$$

(c) For each $\alpha>0$, the operator $(\alpha I-\mathfrak{A})^{-1}$ is nonnegative on the space $C_{0}(K)$ :

$$
f \in C_{0}(K), f(x) \geq 0 \quad \text { on } K \Longrightarrow(\alpha I-\mathfrak{A})^{-1} f(x) \geq 0 \quad \text { on } K .
$$

(d) For each $\alpha>0$, the operator $(\alpha I-\mathfrak{A})^{-1}$ is bounded on the space $C_{0}(K)$ with norm

$$
\left\|(\alpha I-\mathfrak{A})^{-1}\right\| \leq \frac{1}{\alpha} .
$$

(ii) Conversely, if $\mathfrak{A}$ is a linear operator from $C_{0}(K)$ into itself satisfying condition (a) and if there is a constant $\alpha_{0} \geq 0$ such that, for all $\alpha>\alpha_{0}$, conditions (b) through (d) are satisfied, then $\mathfrak{A}$ is the infinitesimal generator of some Feller semigroup $\left\{T_{t}\right\}_{t \geq 0}$ on $K$.

2.3 The spaces $C_{\pi}(\bar{D})$ and $C_{0}(\bar{D})$. First we consider a one-point compactification of the domain $D$. Two points $x$ and $y$ of $\bar{D}$ are said to be equivalent modulo $\partial D$ if either $x=y$ or $x, y \in \partial D$. On the totality $\bar{D} / \partial D$ of equivalence classes, we define the quotient topology induced by the projection $q: \bar{D} \rightarrow \bar{D} / \partial D$. Then it is easy to see that the topological space $\bar{D} / \partial D$ is a one-point compactification of the domain $K=D$ and that the point at infinity $\partial$ corresponds to the boundary $\partial D$, that is,

$$
K_{\partial}=\bar{D} / \partial D, \quad \partial=\partial D .
$$

Furthermore, we have the following assertions:

(i) If $\tilde{u}$ is a continuous function defined on $\bar{D} / \partial D$, then the function $\tilde{u} \circ q$ is continuous on $\bar{D}$ and constant on $\partial D$.

(ii) Conversely, if $u$ is a continuous function defined on $\bar{D}$ and constant on $\partial D$, then it defines a continuous function $\tilde{u}$ on $\bar{D} / \partial D$.

In other words, by letting

$$
C_{\pi}(\bar{D})=\{u \in C(\bar{D}): u \text { is constant on } \partial D\},
$$

we have the following isomorphism:

$$
C\left(K_{\partial}\right) \cong C_{\pi}(\bar{D}) .
$$

On the other hand, if we introduce a closed subspace of $C\left(K_{\partial}\right)$ as

$$
C_{0}(K)=\left\{u \in C\left(K_{\partial}\right): u(\partial)=0\right\},
$$

then we have, by assertion (2.3),

$$
C_{0}(K) \cong C_{0}(\bar{D})=\{u \in C(\bar{D}): u=0 \text { on } \partial D\} .
$$




\section{Proof of Theorems 1, 2 and 3}

The purpose of this section is to prove Theorems 1, 2 and 3, by adapting some results of [9] and [10] to the present context.

3.1 Proof of Theorem 1. (I) First, we start by recalling the following existence and uniqueness theorem for the degenerate Dirichlet problem in the framework of Hölder spaces (see [9, Theorem 3.8]):

Theorem 3.1. Assume that hypothesis (H) is satisfied and that

$$
c(x)<0 \quad \text { on } \bar{D}
$$

and

$$
c^{*}(x):=\sum_{i, j=1}^{N} \frac{\partial^{2} a^{i j}}{\partial x_{i} \partial x_{j}}(x)-\sum_{i=1}^{N} \frac{\partial b^{i}}{\partial x_{i}}(x)+c(x)<0 \quad \text { on } \bar{D} .
$$

Then, for each integer $m \geq 2$, we can find a positive constant $\lambda(m)$ depending on $m$ such that, for each $\lambda \geq \lambda(m)$, the Dirichlet problem

$$
\begin{cases}(A-\lambda) u=f & \text { in } D \\ u=\varphi & \text { on } \partial D\end{cases}
$$

has a unique solution $u$ in $C^{m+\theta}(\bar{D})$ for any $f \in C^{2 m+2+2 \theta}(\bar{D})$ and any $\varphi \in$ $C^{2 m+4+2 \theta}(\partial D)$, with $0<\theta<1$.

The next theorem is an immediate consequence of Theorem 3.1 (see [10, Theorem 4.1]):

Theorem 3.2. If hypothesis (H) is satisfied, then, for each integer $m \geq 2$, we can find a positive constant $\alpha(m)$ depending on $m$ such that, for each $\alpha \geq \alpha(m)$, the Dirichlet problem

$$
\begin{cases}(\alpha-A) u=f & \text { in } D, \\ u=\varphi & \text { on } \partial D\end{cases}
$$

has a unique solution $u \in C^{m+\theta}(\bar{D})$ for any $f \in C^{2 m+2+2 \theta}(\bar{D})$ and any $\varphi \in$ $C^{2 m+4+2 \theta}(\partial D)$, with $0<\theta<1$.

Indeed, it suffices to take a parameter $\alpha>0$ so large that

$$
c^{*}(x)-\alpha=\sum_{i, j=1}^{N} \frac{\partial^{2} a^{i j}}{\partial x_{i} \partial x_{j}}(x)-\sum_{i=1}^{N} \frac{\partial b^{i}}{\partial x_{i}}(x)+c(x)-\alpha<0 \quad \text { on } \bar{D},
$$

and also $\alpha \geq \lambda(m)$.

If we take $m=2$ and $\alpha \geq \alpha(2)$, then Theorem 3.2 allows us to introduce two linear operators $G_{\alpha}^{0}$, called the Green operator, and $H_{\alpha}$, called the harmonic operator, respectively, by the formulas

$$
\begin{gathered}
G_{\alpha}^{0}: C^{6+2 \theta}(\bar{D}) \longrightarrow C^{2+\theta}(\bar{D}) \\
f \longmapsto G_{\alpha}^{0} f
\end{gathered}
$$


and

$$
\begin{gathered}
H_{\alpha}: C^{8+2 \theta}(\partial D) \longrightarrow C^{2+\theta}(\bar{D}) \\
\varphi \longmapsto H_{\alpha} \varphi
\end{gathered}
$$

Here the function $G_{\alpha}^{0} f \in C^{2+\theta}(\bar{D})$ is the unique solution of the problem

$$
\begin{cases}(\alpha-A) G_{\alpha}^{0} f=f & \text { in } D, \\ G_{\alpha}^{0} f=0 & \text { on } \partial D,\end{cases}
$$

and the function $H_{\alpha} \varphi \in C^{2+\theta}(\bar{D})$ is the unique solution of the problem

$$
\begin{cases}(\alpha-A) H_{\alpha} \varphi=0 & \text { in } D, \\ H_{\alpha} \varphi=\varphi & \text { on } \partial D .\end{cases}
$$

(II) Secondly, in order to study problem (D) in the framework of the space of continuous functions, we need the following result (see [10, Theorem 4.2]):

Theorem 3.3. (i) The operator $G_{\alpha}^{0}$ can be uniquely extended to a nonnegative, bounded linear operator from $C(\bar{D})$ into itself, denoted again $G_{\alpha}^{0}$, with norm $\left\|G_{\alpha}^{0}\right\| \leq$ $1 / \alpha$.

(ii) The operator $H_{\alpha}$ can be uniquely extended to a nonnegative, bounded linear operator from $C(\partial D)$ into $C(\bar{D})$, denoted again $H_{\alpha}$, with norm $\left\|H_{\alpha}\right\|=1$.

Moreover we introduce a linear operator

$$
\begin{gathered}
A: C(\bar{D}) \longrightarrow C(\bar{D}) \\
u \longmapsto A u,
\end{gathered}
$$

with domain

$$
D(A)=C^{2}(\bar{D})
$$

Then we know (see [10, Lemma 4.3]) that the operator $A$ has its minimal closed extension $\bar{A}$ in the space $C(\bar{D})$.

The extended operators $G_{\alpha}^{0}: C(\bar{D}) \rightarrow C(\bar{D})$ and $H_{\alpha}: C(\partial D) \rightarrow C(\bar{D})$ still satisfy formulas (3.1) and (3.2) respectively in the following sense (see [10, Lemma 4.4]):

Lemma 3.4. (i) For any $f \in C(\bar{D})$, the function $G_{\alpha}^{0} f$ belongs to the domain $D(\bar{A})$ of $\bar{A}$, and satisfies the conditions

$$
\left\{\begin{array}{l}
(\alpha I-\bar{A}) G_{\alpha}^{0} f=f \\
\left.G_{\alpha}^{0} f\right|_{\partial D}=0
\end{array}\right.
$$

(ii) For any $\varphi \in C(\partial D)$, the function $H_{\alpha} \varphi$ belongs to the domain $D(\bar{A})$, and satisfies the conditions

$$
\left\{\begin{array}{l}
(\alpha I-\bar{A}) H_{\alpha} \varphi=0 \\
\left.H_{\alpha} \varphi\right|_{\partial D}=\varphi .
\end{array}\right.
$$

(iii) Furthermore, every function $u \in D(\bar{A})$ can be written in the form

$$
u=G_{\alpha}^{0}((\alpha I-\bar{A}) u)+H_{\alpha}\left(\left.u\right|_{\partial D}\right) .
$$

(III) Now we are in a position to prove Theorem 1 via the following result: 
Theorem 3.5. Assume that A satisfies hypothesis (H). We define a linear operator

$$
\mathcal{A}: C(\bar{D}) \longrightarrow C(\bar{D})
$$

as follows:

(1) $D(\mathcal{A})=\{u \in D(\bar{A}): \bar{A} u=0$ on $\partial D\}$.

(2) $\mathcal{A} u=\bar{A} u, u \in D(\mathcal{A})$.

Then the operator $\mathcal{A}$ generates a Feller semigroup on $C(\bar{D})$, and the Green operator $G_{\alpha}=(\alpha I-\mathcal{A})^{-1}, \alpha>0$, is given by the formula

$$
G_{\alpha} f=G_{\alpha}^{0} f+\frac{1}{\alpha} H_{\alpha}\left(\left.f\right|_{\partial D}\right), \quad f \in C(\bar{D}) .
$$

Proof. It suffices to verify conditions (a) through (d) of Theorem 2.3, with $C_{0}(K)$ replaced by $C(\bar{D})$, since $C_{0}(K)$ may be identified with $C(K)$ if $K$ is compact. The proof is divided into four steps.

(1) First we verify condition (b). By parts (i) and (ii) of Lemma 3.4, we have, for any $f \in C(\bar{D})$,

$$
G_{\alpha} f \in D(\bar{A}) .
$$

Furthermore, it follows that

$$
\begin{aligned}
\bar{A}\left(G_{\alpha} f\right) & =\bar{A} G_{\alpha}^{0} f+\frac{1}{\alpha} \bar{A} H_{\alpha}\left(\left.f\right|_{\partial D}\right) \\
& =\alpha G_{\alpha}^{0} f-f+H_{\alpha}\left(\left.f\right|_{\partial D}\right),
\end{aligned}
$$

so that

$$
\left.\bar{A}\left(G_{\alpha} f\right)\right|_{\partial D}=-\left.f\right|_{\partial D}+\left.f\right|_{\partial D}=0 .
$$

This proves that $G_{\alpha} f \in D(\mathcal{A})$.

Next we prove that

$$
G_{\alpha}=(\alpha I-\mathcal{A})^{-1}
$$

which verifies condition (b).

Since we have $(\alpha I-\bar{A}) G_{\alpha} f=f$ for all $f \in C(\bar{D})$, in order to prove formula (3.4) it suffices to show the injectivity of $\alpha I-\mathcal{A}$ :

$$
u \in D(\mathcal{A}),(\alpha I-\mathcal{A}) u=0 \Longrightarrow u=0 .
$$

By part (iii) of Lemma 3.4 , we can write $u \in D(\mathcal{A})$ in the form

$$
u=G_{\alpha}^{0}((\alpha-\bar{A}) u)+H_{\alpha}\left(\left.u\right|_{\partial D}\right)=H_{\alpha}\left(\left.u\right|_{\partial D}\right), \quad \alpha>0 .
$$

However, it follows that

$$
0=\left.\bar{A} u\right|_{\partial D}=\left.\bar{A} H_{\alpha}\left(\left.u\right|_{\partial D}\right)\right|_{\partial D}=\alpha\left(\left.u\right|_{\partial D}\right),
$$

so that

$$
\left.u\right|_{\partial D}=0 .
$$


This implies that

$$
u=H_{\alpha}\left(\left.u\right|_{\partial D}\right)=0 \text { in } D .
$$

Summing up, we have proved formula (3.4).

(2) Secondly, by Theorem 3.3 it follows that

$$
f \in C(\bar{D}), f(x) \geq 0 \text { on } \bar{D} \Longrightarrow G_{\alpha}^{0} f(x) \geq 0, H_{\alpha}\left(\left.f\right|_{\partial D}\right)(x) \geq 0 \text { on } \bar{D} \text {. }
$$

This verifies condition (c).

(3) Thirdly, we verify condition (d). If $f \in C(\bar{D})$, then it follows from an application of Lemma 3.4 that

$$
\begin{aligned}
(\alpha-\bar{A}) G_{\alpha} f & =(\alpha-\bar{A}) G_{\alpha}^{0} f+\frac{1}{\alpha}(\alpha-\bar{A}) H_{\alpha}\left(\left.f\right|_{\partial D}\right) \\
& =f \quad \text { in } D
\end{aligned}
$$

and also that

$$
G_{\alpha} f=\frac{1}{\alpha} f \quad \text { on } \partial D
$$

Moreover we remark that

$$
\min _{x \in \bar{D}}(\alpha-c(x)) \geq \alpha
$$

since $c(x) \leq 0$ in $D$.

Therefore, by applying the maximum principle, [9, Theorem 2.3], to the operator $A-\alpha$ with $u:=G_{\alpha} f$ and $g:=(1 / \alpha)\left(\left.f\right|_{\partial D}\right)$ we obtain from [9, inequality (2.4)] that

$$
\left\|G_{\alpha} f\right\| \leq \frac{1}{\alpha}\|f\|
$$

This proves condition $(\mathrm{d})$.

(4) It remains to verify the density of the domain $D(\mathcal{A})$. Since $C^{\infty}(\bar{D})$ is dense in $C(\bar{D})$, we may assume that $f \in C^{\infty}(\bar{D})$. As a consequence of Theorem 3.2, it follows that $G_{\alpha}^{0} f \in C^{2}(\bar{D})$ and $H_{\alpha}\left(\left.f\right|_{\partial D}\right) \in C^{2}(\bar{D})$. Hence we have, by formula $(3.3)$,

$$
G_{\alpha} f \in D(\mathcal{A}) \cap C^{2}(\bar{D}) .
$$

Furthermore, by part (iii) of Lemma 3.4 we can write $f \in C^{\infty}(\bar{D})$ in the form

$$
f=G_{\alpha}^{0}((\alpha-A) f)+H_{\alpha}\left(\left.f\right|_{\partial D}\right)
$$

Hence we have

$$
\begin{aligned}
\alpha G_{\alpha} f-f & =\alpha G_{\alpha}^{0} f+H_{\alpha}\left(\left.f\right|_{\partial D}\right)-G_{\alpha}^{0}((\alpha-A) f)-H_{\alpha}\left(\left.f\right|_{\partial D}\right) \\
& =G_{\alpha}^{0}(A f)
\end{aligned}
$$

However, it follows from an application of part (i) of Theorem 3.3 that

$$
\left\|G_{\alpha}^{0}(A f)\right\| \leq \frac{1}{\alpha}\|A f\|
$$


Therefore, we have proved that

and so

$$
\left\|\alpha G_{\alpha} f-f\right\|=\left\|G_{\alpha}^{0}(A f)\right\| \leq \frac{1}{\alpha}\|A f\|,
$$

$$
\lim _{\alpha \rightarrow+\infty} \alpha G_{\alpha} f=f \quad \text { in } C(\bar{D}) .
$$

This verifies condition (a), and Theorem 3.5 is proved.

(IV) Finally, we can prove that

$$
D(\mathcal{A})=\{u \in C(\bar{D}): A u \in C(\bar{D}), A u=0 \text { on } \partial D\},
$$

where $A u$ is taken in the sense of distributions.

Indeed, let $u$ be an arbitrary function in $C(\bar{D})$ such that $A u \in C(\bar{D})$ and $A u=0$ on $\partial D$. Then we obtain that, for any $\alpha>0$ (sufficiently large), the function

$$
w=u-G_{\alpha}((\alpha-A) u)
$$

satisfies the conditions

$$
(\alpha-A) w=0 \quad \text { in } D,
$$

and

$$
w=u-\frac{1}{\alpha}(\alpha u-A u)=0 \quad \text { on } \partial D .
$$

Hence it follows from an application of the uniqueness theorem for problem (D), [9, Theorem 2.6], that $w=0$. By formula (3.4), this implies that

$$
u=G_{\alpha}((\alpha-A) u) \in D(\mathcal{A}) .
$$

Now the proof of Theorem 1 is complete.

3.2 Proof of Theorem 2. For any $f \in C_{\pi}(\bar{D})$, the function

$$
u=G_{\alpha} f=G_{\alpha}^{0} f+\frac{1}{\alpha} H_{\alpha}\left(\left.f\right|_{\partial D}\right)
$$

satisfies the conditions

$$
\begin{cases}(\alpha-A) u=f & \text { in } D, \\ u=\frac{1}{\alpha} f & \text { on } \partial D,\end{cases}
$$

and

$$
A u=\alpha u-f=0 \quad \text { on } \partial D .
$$

This proves that $u \in D(\mathcal{P})$, since $f$ is constant on $\partial D$.

Therefore, Theorem 2 is an immediate consequence of Theorem 1.

3.3 Proof of Theorem 3. In a similar way, for any $f \in C_{0}(\bar{D})$, the function

$$
u=G_{\alpha}^{0} f
$$

satisfies the conditions

$$
\begin{cases}(\alpha-A) u=f & \text { in } D \\ u=0 & \text { on } \partial D\end{cases}
$$

and

$$
A u=\alpha u-f=0 \quad \text { on } \partial D,
$$

which proves that $u \in D(\mathcal{C})$. Hence, Theorem 3 follows from an application of Theorem 1. 


\section{References}

[1] Clément, Ph. and C. A. Timmermanns, On $C_{0}$-semigroups generated by differential operators satisfying Ventcel' boundary conditions, Indag. Math. 89 (1986), 379-387.

[2] Dynkin, E. B., "Markov processes" I, II, Springer-Verlag, Berlin, Göttingen, Heidelberg, 1965.

[3] Favini, A. and S. Romanelli, Analytic semigroups on $C[0,1]$ generated by some classes of second order differential operators, Semigroup Forum 56 (1998), 362-372.

[4] Fichera, G., Sulla equazioni differenziali lineari ellittico-paraboliche del secondo ordine, Atti Accad. Naz. Lincei Mem. 5 (1956), 1-30.

[5] Lamperti, J., "Stochastic processes", Springer-Verlag, New York, Heidelberg, Berlin, 1977.

[6] Oleı̆nik, O. A. and E. V. Radkevič, "Second order equations with nonnegative characteristic form", Amer. Math. Soc., Providence, Rhode Island and Plenum Press, New York, 1973.

[7] Stroock, D. W. and S. R. S. Varadhan, On degenerate elliptic-parabolic operators of second order and their associated diffusions, Comm. Pure Appl. Math. 25 (1972), 651-713.

[8] Taira, K., "Diffusion processes and partial differential equations", Academic Press, San Diego, New York, London, Tokyo, 1988.

[9] Taira, K., On the existence of Feller semigroups with Dirichlet condition, Tsukuba J. Math. 17 (1993), 377-427.

[10] Taira, K., On the existence of Feller semigroups with boundary conditions II, J. Functional Analysis 129 (1995), 108-131.

Institute of Mathematics, University of Tsukuba, Tsukuba 305-8571, JAPAN

E-mail address: taira@math.tsukuba.ac.jp

Università di Bologna, Dipartimento di Matematica, 40126 Bologna, Italy

E-mail address: favini@dm.unibo.it

Università di Bari, Dipartimento di Matematica, 70125 Bari, Italy

E-mail address: romans@pascal.dm.uniba.it 